\title{
A likelihood ratio approach for identifying three-quarter siblings in genetic databases
}

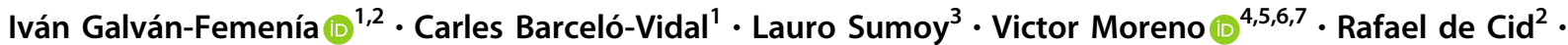 \\ Jan Graffelman $\mathbb{1}^{8,9}$
}

Received: 26 October 2020 / Revised: 4 November 2020 / Accepted: 16 November 2020 / Published online: 15 January 2021

(c) The Author(s) 2021. This article is published with open access

\begin{abstract}
The detection of family relationships in genetic databases is of interest in various scientific disciplines such as genetic epidemiology, population and conservation genetics, forensic science, and genealogical research. Nowadays, screening genetic databases for related individuals forms an important aspect of standard quality control procedures. Relatedness research is usually based on an allele sharing analysis of identity by state (IBS) or identity by descent (IBD) alleles. Existing IBS/IBD methods mainly aim to identify first-degree relationships (parent-offspring or full siblings) and second degree (half-siblings, avuncular, or grandparent-grandchild) pairs. Little attention has been paid to the detection of in-between first and second-degree relationships such as three-quarter siblings (3/4S) who share fewer alleles than first-degree relationships but more alleles than second-degree relationships. With the progressively increasing sample sizes used in genetic research, it becomes more likely that such relationships are present in the database under study. In this paper, we extend existing likelihood ratio (LR) methodology to accurately infer the existence of $3 / 4 \mathrm{~S}$, distinguishing them from full siblings and second-degree relatives. We use bootstrap confidence intervals to express uncertainty in the LRs. Our proposal accounts for linkage disequilibrium (LD) by using marker pruning, and we validate our methodology with a pedigree-based simulation study accounting for both LD and recombination. An empirical genome-wide array data set from the GCAT Genomes for Life cohort project is used to illustrate the method.
\end{abstract}

Associate editor: Armando Caballero

Supplementary information The online version of this article (https:// doi.org/10.1038/s41437-020-00392-8) contains supplementary material, which is available to authorized users.

Rafael de Cid

rdecid@igtp.cat

$\bowtie$ Jan Graffelman

jan.graffelman@upc.edu

1 Department of Computer Science, Applied Mathematics and Statistics, Universitat de Girona, Girona, Spain

2 Genomes For Life - GCAT lab, Institute for Health Science Research Germans Trias i Pujol (IGTP), Can Ruti Campus, Badalona, Barcelona, Spain

3 High Content Genomics and Bioinformatics Unit, Institute for Health Science Research Germans Trias i Pujol (IGTP), Can Ruti Campus, Badalona, Barcelona, Spain

\section{Introduction}

The detection of related individuals in genetic databases is of great interest in various areas of genetic research. Most obviously, it is of interest in forensic studies aiming at identifying relationships between individuals such as paternity tests (Evett and Weir, 1998) or sibling tests

4 Oncology Data Analytics Program, Catalan Institute of Oncology (ICO), Badalona, Spain

5 ONCOBELL Program, Bellvitge Biomedical Research Institute (IDIBELL), Barcelona, Spain

6 Consortium for Biomedical Research in Epidemiology and Public Health (CIBERESP), Madrid, Spain

7 Department of Clinical Sciences, University of Barcelona, Barcelona, Spain

8 Department of Statistics and Operations Research, Universitat Politècnica de Catalunya, Barcelona, Spain

9 Department of Biostatistics, University of Washington, Seattle, WA, USA 
Table 1 Degree of relationship (R), kinship coefficient $(\phi)$, and probability of sharing zero, one or two alleles identical by descent $\left(k_{0}\right.$, $\left.k_{1}, k_{2}\right)$.

\begin{tabular}{|c|c|c|c|c|c|}
\hline \multirow[b]{2}{*}{ Type of relative } & \multirow[b]{2}{*}{$\mathrm{R}$} & \multirow[b]{2}{*}{$\phi$} & \multicolumn{3}{|c|}{$\begin{array}{l}\text { Probability of } \\
\text { IBD sharing }\end{array}$} \\
\hline & & & $k_{0}$ & $k_{1}$ & $k_{2}$ \\
\hline Monozygotic twins (MZ) & 0 & $1 / 2$ & 0 & 0 & 1 \\
\hline Parent-offspring (PO) & 1 & $1 / 4$ & 0 & 1 & 0 \\
\hline Full siblings (FS) & 1 & $1 / 4$ & $1 / 4$ & $1 / 2$ & $1 / 4$ \\
\hline Three-quarter siblings (3/4S) & - & $3 / 16$ & $3 / 8$ & $1 / 2$ & $1 / 8$ \\
\hline $\begin{array}{l}\text { Half-siblings/grandchild-grandparent/niece/ } \\
\text { nephew-uncle/aunt (2nd) }\end{array}$ & 2 & $1 / 8$ & $1 / 2$ & $1 / 2$ & 0 \\
\hline First cousins $(\mathrm{FC})$ & 3 & $1 / 16$ & $3 / 4$ & $1 / 4$ & 0 \\
\hline Unrelated (UN) & $\infty$ & 0 & 1 & 0 & 0 \\
\hline
\end{tabular}

(Mo et al., 2016, Wang, 2004). Good high-resolution techniques for detecting related individuals are also of interest for genealogical research on family reconstruction (Staples et al., 2014). In conservation genetics, careful selection of unrelated individuals for breeding programs is needed (Oliehoek et al., 2006), requiring the estimation of pairwise genetic relationships. In genome-wide association studies (GWAS) that have become popular during the past two decades (Visscher et al., 2017), standard quality control filters are applied prior to genetic association analysis. The presence of cryptic relatedness violates the assumption of independent individuals in association modeling. For this reason, removing related individuals in the genetic database prior to the GWAS analysis is a common quality control step (Anderson et al., 2010).

Many methods for relatedness research are described in the literature. Most of them are based on the principle of allele sharing. Two individuals can share 0,1 , or 2 alleles for a diploid genetic marker. These alleles can be identical by state (IBS) or identical by descent (IBD). A scatterplot of the mean $\left(\bar{x}_{I B S}\right)$ and standard deviation $\left(s_{I B S}\right)$ of the number of IBS alleles over variants can be used to identify related pairs (Abecasis et al., 2001). Alternatively, a scatterplot of the proportions of sharing 0,1 , or 2 IBS alleles $\left(p_{0}, p_{1}, p_{2}\right)$ is also often used to detect related pairs (Rosenberg, 2006). In genetic studies, the probabilities of sharing 0,1 , and 2 IBD alleles $\left(k_{0}\right.$, $k_{1}, k_{2}$ ) can be estimated and used for relationship inference, since their theoretically expected values are known for the standard relationships (see Table 1). For example, parent-offspring pairs have $\left(k_{0}, k_{1}, k_{2}\right)=(0,1,0)$ and full siblings have $\left(k_{0}, k_{1}, k_{2}\right)=(0.25,0.50,0.25)$. For a given pair of individuals, these probabilities can be estimated by maximum likelihood (Milligan, 2003, Thompson, 1975, 1991), by the method of moments (Purcell et al., 2007) or with robust estimators (Manichaikul et al., 2010). From these probabilities, the kinship coefficient, defined as $\phi=k_{1} / 4+k_{2} / 2$, can be obtained. The kinship coefficient can be used to remove individuals with first degree (parent-offspring (PO) or full siblings (FS)) and second-degree relationships (half-siblings, avuncular or grandparent-grandchild) by retaining only pairs with $\phi<1 / 16$. In addition, third-degree relationships (first cousins (FC)) can be eliminated by retaining only pairs with $\phi$ $<1 / 32$ (Anderson et al., 2010). All these methods have in common that the inference of the family relationships is based on the judgment of the analyst of the point estimates ( $\left.\hat{k}_{0}, \hat{k}_{1}, \hat{k}_{2}, \hat{\phi}\right)$ or of a graphical representation $\left(\left(\bar{x}_{I B S}, s_{I B S}\right),\left(p_{0}\right.\right.$, $\left.p_{1}, p_{2}\right)$ or $\left.\left(\hat{k}_{0}, \hat{k}_{1}, \hat{k}_{2}\right)\right)$ (Galvan-Femenia et al., 2017).

The sample size used in genetic studies, GWAS in particular, is progressively increasing owing to large human sequencing projects that involve genetic data from hundreds of thousands of individuals such as UK Biobank (Bycroft et al., 2018), gnomAD (Karczewski et al., 2020), TOPMed (Taliun et al., 2019), and DiscovEHR (Staples et al., 2018) among others. With such large databases, it becomes increasingly likely that in-between 1 st and 2 nd degree, and in-between 2nd and 3rd-degree relationships are found. Such in-between relationships are mostly ignored in a relatedness analysis, which typically mostly focus on 1st, 2nd, and 3rd-degree relationships. In this paper, we therefore develop a likelihood ratio (LR) approach that will allow us to identify three-quarter siblings (3/4S), a family relationship whose individuals share fewer alleles than 1st-degree relationships but more alleles than 2nd-degree relatives (Table 1). A 3/4S pair has one common parent, whereas their unshared parents have a first-degree relationship (FS or PO; see Graffelman et al. 2019 Fig. S2). The IBD probabilities for $3 / 4 \mathrm{~S}$ are $\left(k_{0}, k_{1}\right.$, $\left.k_{2}\right)=(3 / 8,1 / 2,1 / 8)$ and their kinship coefficient is $\phi=3 /$ 16. A detailed derivation of these probabilities is shown in Appendix A. A 3/4S relationship is not very common, but is more likely to be present in GWAS studies with everincreasing sample sizes. The $3 / 4 \mathrm{~S}$ relationship has received very little attention in the literature, and the aim of this paper is to develop tools that distinguish $3 / 4 \mathrm{~S}$ from full siblings and second-degree relatives.

The remainder of this paper is structured as follows. Section "Methods and materials" develops a LR approach for identifying three-quarter siblings. Section "Simulations" evaluates the LR approach in a simulation study. Section "Case study" illustrates our approach with genome-wide SNP array data from the GCAT Genomes for Life project cohort. Finally, we end the article with a discussion of the proposed methodology.

\section{Methods and materials}

\section{Overview of the likelihood of a relationship}

A detailed derivation of the likelihood of having a given relationship is given by Wagner et al. (2006). In brief, let $n$ 
Table 2 Possible pairs of biallelic genotypes and the probability of each pair given the number of alleles identical by descent $(m)$.

\begin{tabular}{llll}
\hline$G_{1} / G_{2}$ & $m=0$ & $m=1$ & $m=2$ \\
\hline$A A / A A$ & $p^{4}$ & $p^{3}$ & $p^{2}$ \\
$A A / A B$ & $2 p^{3} q$ & $p^{2} q$ & 0 \\
$A A / B B$ & $p^{2} q^{2}$ & 0 & 0 \\
$A B / A B$ & $4 p^{2} q^{2}$ & $p q$ & $2 p q$ \\
\hline
\end{tabular}

We assume that the order of the genotypes is irrelevant, i.e., the probabilities for $G_{1} / G_{2}$ and $G_{2} / G_{1}$ are the same.

be the number of individuals in a non-inbred homogeneous population and assuming absence of population structure. We consider biallelic genetic variants with alleles $A$ and $B$ having allele frequencies $p$ and $q$, respectively. Let $G_{1} / G_{2}$ be the genotypes for a pair of individuals, let $k_{m}$ with $m=0,1$, 2 be their IBD probabilities (shown in Table 1) and let $R$ be their family relationship. Then, the probability of observing $G_{1} / G_{2}$, given $R$ is:

$$
\begin{aligned}
P\left(G_{1} / G_{2} \mid R\right)= & P\left(G_{1} / G_{2} \mid m=0\right) k_{0} \\
& +P\left(G_{1} / G_{2} \mid m=1\right) k_{1} \\
& +P\left(G_{1} / G_{2} \mid m=2\right) k_{2} .
\end{aligned}
$$

The terms $P\left(G_{1} / G_{2} \mid m=0\right), P\left(G_{1} / G_{2} \mid m=1\right)$ and $P\left(G_{1} /\right.$ $G_{2} \mid m=2$ ) are the probabilities of observing each pair of genotypes given the number of IBD alleles (Table 2).

\section{The LR approach for identifying three-quarter siblings}

The LR approach has been widely used for relatedness research during the last decades (Boehnke and Cox, 1997, Heinrich et al., 2016, Katki et al., 2010, Kling and Tillmar, 2019, Thompson, 1986, Weir et al., 2006). In brief, the LR approach is based on the contrast of two hypotheses, one in the numerator, $H_{i}$; and the other one in the denominator, $H_{j}$. The larger the LR, the more plausible is $H_{i}$; whereas the smaller the LR, the more plausible is $H_{j}$. For relatedness research, we consider the ratio of the probabilities from Eq. 1 according to the hypothesis of the $R_{i}$ and $R_{j}$ relationships. That is:

$$
L R\left(R_{i}, R_{j} \mid G_{1} / G_{2}\right)=\frac{P\left(G_{1} / G_{2} \mid R_{i}\right)}{P\left(G_{1} / G_{2} \mid R_{j}\right)}
$$

Here we consider the FS, 3/4S, 2nd, and unrelated (UN) relationships and calculate three LR having FS, 3/4S, or 2nd in the numerator and having the UN relationship in the denominator. The common denominator makes the LR values comparable in order to distinguish $3 / 4 \mathrm{~S}$ from $\mathrm{FS}$ and 2nd degree. The inference of relatedness for each pair of individuals is based on the largest $\mathrm{LR}$ value in the $\mathrm{FS} \sim \mathrm{UN}$, $3 / 4 \mathrm{~S} \sim \mathrm{UN}$, and 2 nd $\sim \mathrm{UN}$ ratios. The LRs are shown in
Table 3 Likelihood ratio (LR) for relatedness research for biallelic SNPs.

\begin{tabular}{lllll}
\hline $\mathrm{LR}$ & $A A / A A$ & $A A / A B$ & $A B / A B$ & $A A / B B$ \\
\hline $\mathrm{PO} \sim \mathrm{UN}$ & $\frac{1}{p}$ & $\frac{1}{2 p}$ & $\frac{1}{4 p q}$ & $\frac{e}{p^{2} q^{2}}$ \\
$\mathrm{FS} \sim \mathrm{UN}$ & $\frac{1}{4}+\frac{1}{2 p}+\frac{1}{(2 p)^{2}}$ & $\frac{1}{4}+\frac{1}{4 p}$ & $\frac{1}{4}+\frac{1}{4 p q}$ & $\frac{1}{4}$ \\
$3 / 4 \mathrm{~S} \sim \mathrm{UN}$ & $\frac{3}{8}+\frac{1}{2 p}+\frac{1}{8 p^{2}}$ & $\frac{3}{8}+\frac{1}{4 p}$ & $\frac{3}{8}+\frac{3}{16 p q}$ & $\frac{3}{8}$ \\
2nd $\sim \mathrm{UN}$ & $\frac{1}{2}+\frac{1}{2 p}$ & $\frac{1}{2}+\frac{1}{4 p}$ & $\frac{1}{2}+\frac{1}{8 p q}$ & $\frac{1}{2}$ \\
$\mathrm{FC} \sim \mathrm{UN}$ & $\frac{3}{4}+\frac{1}{2 p}$ & $\frac{3}{4}+\frac{1}{4 p}$ & $\frac{3}{4}+\frac{1}{16 p q}$ & $\frac{3}{4}$ \\
\hline
\end{tabular}

The considered LR are PO, FS, 3/4S, 2nd, or FC relationships in the numerator and the UN relationship in the denominator. The LR values depend on the observed genotypes of a pair of individuals and the allele frequencies $p$ and $q$ of the population under study. The $e$ parameter is used to account for genotype errors and de novo mutations if the genotype combination does not occur (Heinrich et al., 2016). We assume that the order of the genotypes is irrelevant, i.e., the LR for $G_{1} / G_{2}$ and $G_{2} / G_{1}$ is the same.

Table 3, depending on the observed genotypes of a pair of individuals. Most of these ratios are derived in Heinrich et al. (2016), and the new results for $3 / 4 \mathrm{~S}$ are shown in Appendix $\mathrm{B}$. The $e$ parameter from the $\mathrm{PO} \sim \mathrm{UN}$ ratio in Table 3 is a small number (i.e., 0.001) used to account for genotype errors and de novo mutations if the genotype combination does not occur. In this way, the LR cannot be zero. For $S$ biallelic SNPs, the LR can be obtained by multiplying the $L R_{s}$ across independent markers and by dividing by the number of SNPs. It is convenient to work in a logarithmic scale such that:

$$
\begin{aligned}
\log _{10}(L R) & =\frac{1}{S} \log _{10}\left(\prod_{s=1}^{S} L R_{S}\left(R_{i}, R_{j} \mid G_{1} / G_{2}\right)\right) \\
& =\frac{1}{S} \sum_{s=1}^{S} \log _{10}\left(L R_{S}\left(R_{i}, R_{j} \mid G_{1} / G_{2}\right)\right),
\end{aligned}
$$

which corresponds to the logarithm of the geometric mean of the LRs. Obtained LRs are subject to uncertainty. To assess this uncertainty, we propose to apply bootstrap resampling (Efron and Tibshirani, 1994). This allows the construction of $95 \%$ bootstrap confidence intervals for the LRs, which are helpful to assess which relationship is the most likely one for a given pair.

\section{Materials}

We test our method for detecting $3 / 4 \mathrm{~S}$ with data from the GCAT Genomes for Life cohort project (Obón-Santacana et al., 2018). In brief, the GCAT project is a prospective study that includes $\sim 20 \mathrm{~K}$ participants recruited from the general population of Catalonia, a Western Mediterranean region in the Northeast of Spain. A subset of 5459 participants was genotyped using the Infinium Expanded MultiEthnic Genotyping Array (MEGAEx) (ILLUMINA, San 
Diego, California, USA). In the present work, we consider 5075 GCAT participants of Caucasian ancestry and 756,003 SNPs that passed strict quality control (Galvan-Femenia et al., 2018). A previous relatedness research analysis of this dataset reported $63 \mathrm{FS}$, eight $3 / 4 \mathrm{~S}$, and 12 2nd-degree candidate pairs (Graffelman et al., 2019).

\section{Simulations}

In this section, we evaluate the likelihood ratio approach to distinguish $3 / 4 \mathrm{~S}$ from FS and 2nd relationships by using simulated data. Pedigrees were simulated from the genetic data of the individuals of the GCAT project, using the pedsim method of Caballero et al. (2019). We apply this method in order to account for recombination by using sexspecific genetic maps (Bherer et al., 2017) and also a crossover interference model (Campbell et al., 2015). The simulations were carried out as follows. First, we identified 4147 potentially unrelated individuals with kinship coefficient $<0.025$. From these individuals, we retained 537,488 autosomal SNPs with minor allele frequency (MAF) $>0.01$, Hardy-Weinberg exact mid $p$ value $>0.05$ (Graffelman and Moreno, 2013) and missing call rate zero. Genotypes of the unrelated individuals were phased with SHAPEIT4 (Delaneau et al., 2019) and were used as input for the ped-sim method. Then, we simulated 500 pedigrees containing one FS pair and 500 pedigrees containing one $3 / 4 \mathrm{~S}$ pair. In total, we used 3000 random GCAT individuals as founders to generate 3000 artificial individuals. The number of simulated related pairs were 4,000 PO, $500 \mathrm{FS}, 5003 / 4 \mathrm{~S}$ and 3,500 2nd degree from a total of $17,997,000$ of pairs. To estimate the IBD probabilities and the kinship coefficient for these simulated pairs we used 27,087 SNPs obtained by retaining variants with MAF $>0.40$ and by LD pruning, requiring markers to have low pairwise correlation $\left(r^{2}<0.20\right)$.

Figure 1 shows the $\left(\hat{k}_{0}, \hat{k}_{1}\right)$-plot for these simulated pairs of individuals. The IBD probabilities were estimated with the PLINK software (Purcell et al., 2007). As expected, the estimated IBD probabilities are close to the expected theoretical values from Table 1 for most pairs of individuals. In Fig. 1, the 3/4S relationships show good separation from 2nd-degree relationships but mix to some extent with FS pairs. Estimated IBD probabilities appear to be centered on their expected values for FS, 3/4S, and 2nd-degree pairs, and have larger variance then $\mathrm{PO}$ and UN pairs. The discriminative power of our method crucially depends on the variance of these estimated probabilities (Hill and Weir, 2011).

Boxplots of the kinship estimator recently proposed by Goudet \& Weir (Goudet et al. (2018), Weir and Goudet (2017)) shown in Fig. 2 clearly show a difference in median

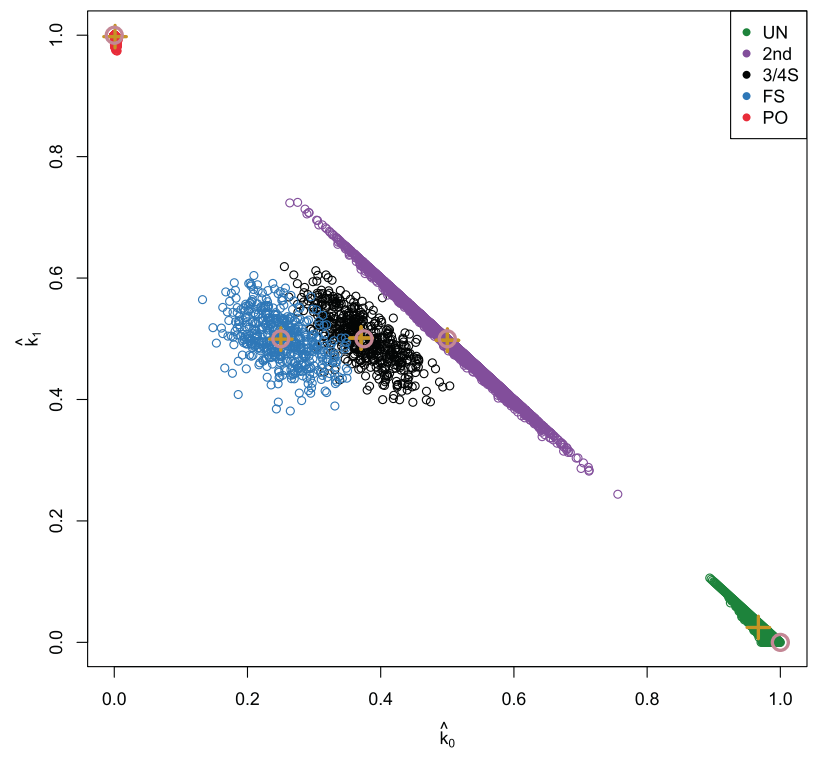

Fig. $1\left(\hat{k}_{0}, \hat{k}_{1}\right)$-plot of $\sim 18$ million pairs of simulated individuals using 27,087 SNPs. UN: unrelated; 2nd: second-degree relationships; 3/4S: three-quarter siblings. FS: full siblings; PO: parent-offspring. Brown open dots represent theoretical IBD probabilities; brown + signs the average of the corresponding group.

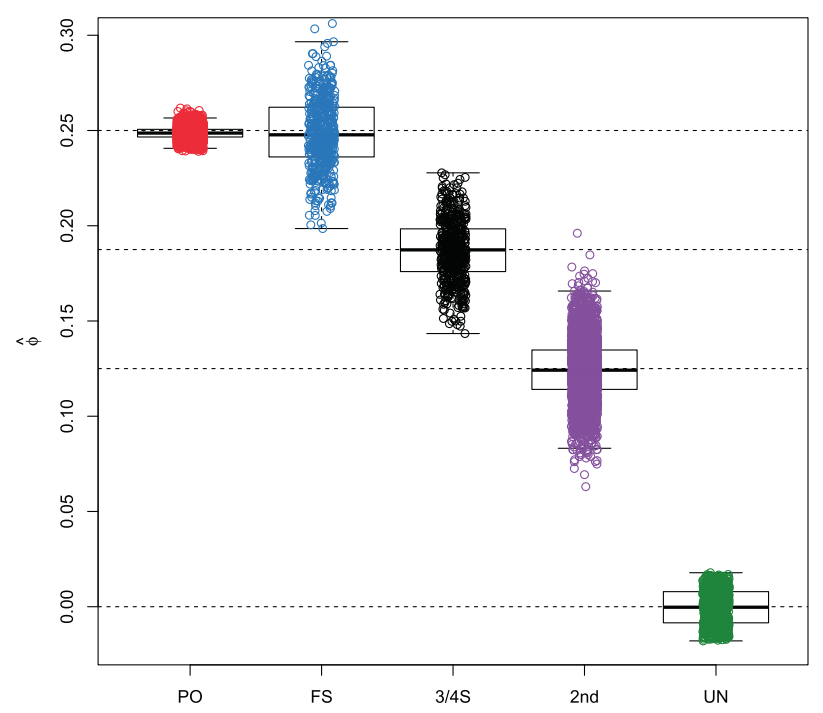

Fig. 2 Boxplot of kinship estimates of $\sim 18$ million pairs of simulated individuals using 27,087 SNPs.

for $3 / 4 \mathrm{~S}$ and 1 st- and 2 nd-degree relationships, though the distribution of the kinship coefficient of the 3/4S overlaps with those of 1 st and 2nd-degree pairs. Also, kinship coefficients can be identical for different relationships, as is the case for PO and FS. Therefore, according to Eq. (3), we calculate the FS $\sim \mathrm{UN}, 3 / 4 \mathrm{~S} \sim \mathrm{UN}$, and 2nd $\sim \mathrm{UN}$ likelihood ratios for 500 2nd, 500 3/4S, and $500 \mathrm{FS}$ simulated pairs. Figure 3 shows that FS pairs mostly have the largest LR values in the FS $\sim \mathrm{UN}$ ratio, $3 / 4 \mathrm{~S}$ pairs mostly have the largest LR values in the $3 / 4 \mathrm{~S} \sim \mathrm{UN}$ ratio and 2 nd-degree 


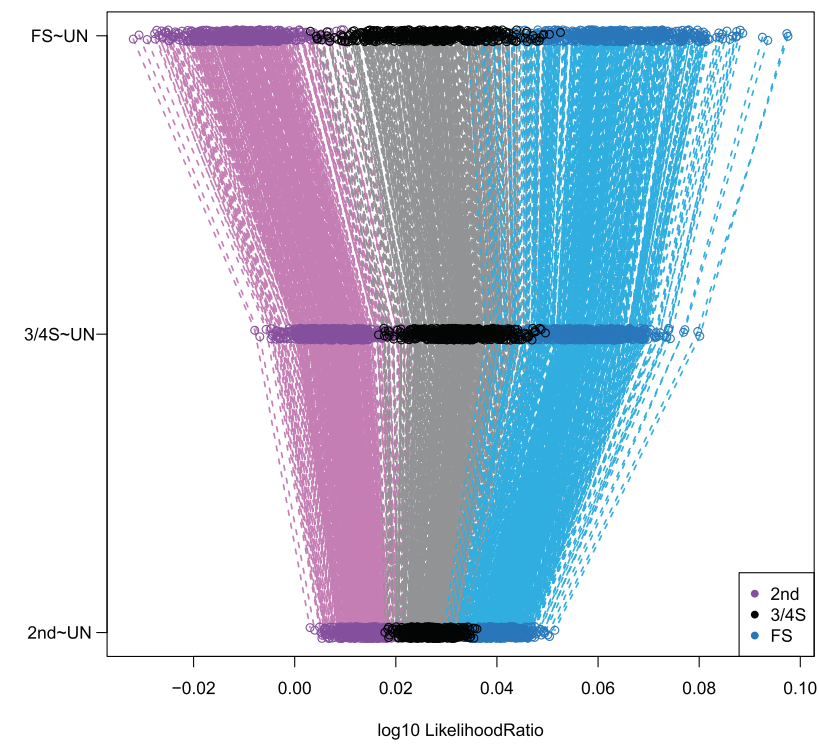

Fig. 3 Log10 likelihood ratio approach of the simulated 2nd, 3/4S, and FS pairs (500 for each relationship) using 27,087 SNPs. Note the larger than sign shaped ("> ") pattern (gray dashed lines) for most 3/4S pairs.

pairs mostly have largest LR in the 2nd $\sim$ UN. Note the plotted data profile shaped in a "greater-than" sign (">") pattern suggesting the inference of $3 / 4 \mathrm{~S}$ for most $3 / 4 \mathrm{~S}$ pairs. In fact, the correct classification rate of the LR approach for the 2nd, $3 / 4 \mathrm{~S}$ and FS simulated pairs is $500 / 500=1,479 /$ $500=0.958$ and $475 / 500=0.95$, respectively. When comparing the correct classification rate of the LR approach with the LR-kinbiplot approach (Graffelman et al., 2019) based on $500 \mathrm{FS}, 5003 / 4 \mathrm{~S}, 3,500$ 2nd, and 5,000 UN simulated pairs (Fig. S1), we observe slightly lower classification rates for $3 / 4 \mathrm{~S} \quad(478 / 500=0.956)$ and $\mathrm{FS}$ $(468 / 500=0.936)$ using linear discriminant analysis and slightly better classification rates for $3 / 4 \mathrm{~S}(481 / 500=$ $0.962)$ and FS $(483 / 500=0.966)$ when using quadratic discriminant analysis as a predictive model. These simulations show the proposed LR approach to be useful for distinguishing 3/4S relationships from FS and 2nd-degree relationships, and to have similar performance to the previously proposed LR-kinbiplot approach.

\section{Case study}

In this section, we apply the likelihood ratio approach to genome-wide SNP array data from the aforementioned GCAT project. Graffelman et al. (2019, Table 5 and Fig. 7) suggested this database to contain eight $3 / 4 \mathrm{~S}$ pairs using a log-ratio biplot approach combined with discriminant analysis (LR-kinbiplot). Figures 4 and 5 show the $\left(\hat{k}_{0}, \hat{k}_{1}\right)$-plot and boxplots of kinship estimates of the GCAT data. The

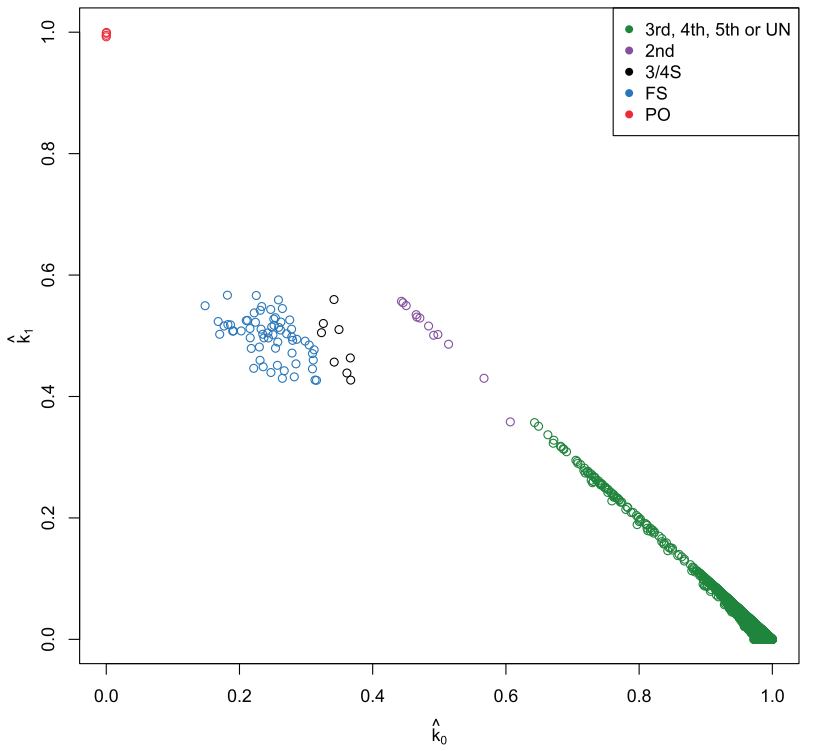

Fig. $4\left(\hat{k}_{0}, \hat{k}_{1}\right)$-plot of the GCAT cohort for $\mathbf{5 0 7 5}$ individuals and 26,006 SNPs (MAF > 0.40, LD-pruned, HWE exact mid $p$ value $>$ $\mathbf{0 . 0 5}$, and missing call rate 0 ). $3 \mathrm{rd}$, 4 th, 5 th, or UN: third, fourth, fifthdegree relationships or unrelated; 2nd: second-degree relationships; 3/ 4S: three-quarter siblings; FS: full siblings; PO: parent-offspring.

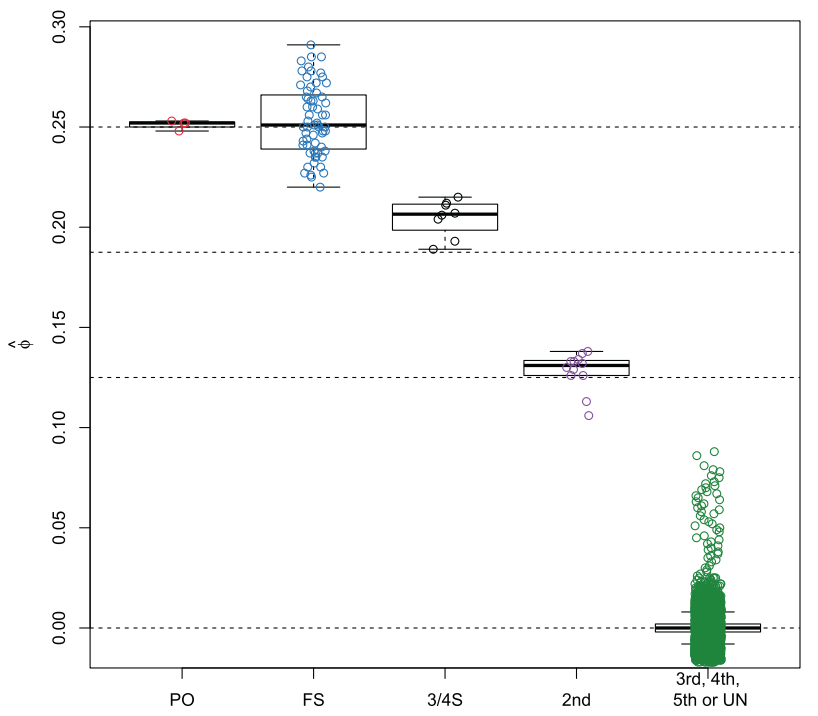

Fig. 5 Boxplot of kinship estimates of the GCAT cohort for 5,075 individuals and 26,006 SNPs (MAF > 0.40, LD-pruned, HWE exact mid $p$ value $>0.05$, and missing call rate 0 ).

IBD probabilities were estimated with the PLINK software, whereas the kinship coefficient was estimated by the estimator proposed by Weir and Goudet (2017). The colors for the FS, 3/4S, and 2nd-degree pairs in both Figures were assigned according to the relationship inferred by the LR approach. Figure 4 shows, like the simulations, a larger variance for FS pairs, and smaller variances for $\mathrm{PO}$ and UN pairs. 


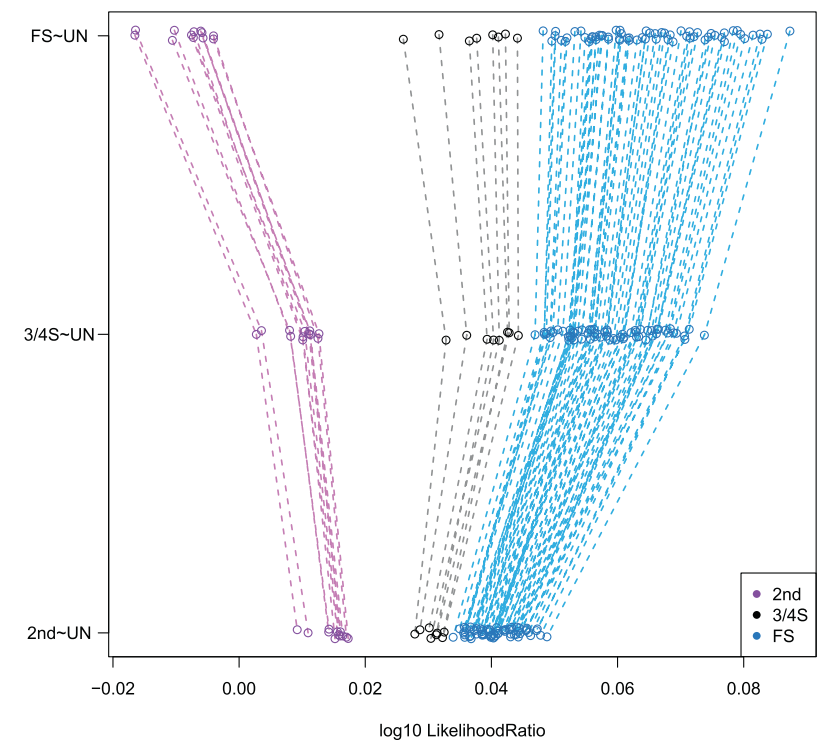

Fig. 6 Log 10 likelihood ratio approach of the presumably 2nd, 3/4S, and FS pairs from the GCAT cohort using 26,006 SNPs (MAF $>0.40$, LD-pruned, HWE exact mid $p$ value $>0.05$, and missing call rate 0 ).

Figure 6 shows the LR ratio values for the three relationships (FS $\sim \mathrm{UN}, 3 / 4 \mathrm{~S} \sim \mathrm{UN}$ and 2 nd $\sim \mathrm{UN}$ ratios) on the horizontal axis, for the presumably FS, 3/4S and 2nd pairs from the GCAT project. The LR analysis reveals eight 3/4S pairs (black color) that have the 'greater-than' sign (">") shaped pattern, because the largest LR values are obtained for the $3 / 4 \mathrm{~S} \sim \mathrm{UN}$ ratio. All inferred FS pairs (blue color) have a monotonously increasing shaped pattern (" $\% ")$ since the largest LR values are obtained for the FS UN ratio; and all 2nddegree pairs have a monotonously decreasing pattern ("l") since the largest LR values are obtained for the 2 nd $\sim \mathrm{UN}$ ratio. Table 4 shows the LR values for each pair which confirm that there are eight $3 / 4 \mathrm{~S}$ pairs in concordance with the LR-kinbiplot approach. We used bootrapping to assess the amount of uncertainty in the LRs. The bootstrap distribution of the LR for the eight hypothesized $3 / 4 \mathrm{~S}$ pairs is shown in Fig. 7. This plot shows seven pairs having the entire bootstrap distributions for the two relationships completely separated, and these pairs therefore clearly do not correspond to FS pairs. For one pair (20) the $3 / 4 \mathrm{~S}$ relationship is most likely, for having on average the largest LR; however, given the overlap of the two distributions, the evidence for a $3 / 4 \mathrm{~S}$ relationship is less compelling for this pair.

\section{Discussion}

In this paper, we show that the likelihood ratio approach is useful for distinguishing three-quarter siblings from FS and 2nd-degree relationships. Figure 4 shows that in a standard $\left(\hat{k}_{0}, \hat{k}_{1}\right)$-plot, $3 / 4 \mathrm{~S}$ can easily go unnoticed as FS pairs. The
LR approach can be of great help to detect such cases. The LR approach developed in this paper confirmed eight 3/4S pairs previously uncovered by a log-ratio biplot (LR-kinbiplot) approach (Graffelman et al., 2019) for genome-wide SNP array data from the GCAT cohort. The assessment of the precise relationship of a pair based on the numerical values of the LRs, or on a plot of the LRs, ignores the uncertainty in these statistics. We found bootstrap procedures to be extremely useful for quantifying this uncertainty, and consider it to be an invaluable tool for the sensible interpretation of the pairwise LR statistics.

The estimated relationships for the GCAT cohort were to some extent confirmed by an analysis of the surnames of the participants, respecting their privacy. In Spain, people have a double surname, usually the first from the father and the second from the mother. This implies that FS and 3/4S pairs share two surnames, whereas 2nd-degree relationships share only one. All identified $3 / 4 \mathrm{~S}$ pairs were confirmed to share two surnames, supporting that these pairs are not 2nd degree.

The proposed LR approach multiplies the likelihoods over loci, under the assumption of independence. The existence of LD between variants violates this assumption. In order to approximately meet the requirement of independence, LD pruning of neighboring variants in a window is therefore recommended (Kling and Tillmar, 2019). This pruning can be done in PLINK (Purcell et al., 2007) or with other software (Calus and Vandenplas, 2018). A future improvement of the LR approach could use Markov chain algorithms (Abecasis and Wigginton, 2005, Kling et al., 2015) that allow efficient likelihood computations on blocks of tightly linked markers.

The LR approach developed in this paper assumes known allele frequencies and non-inbred individuals. The first assumption seems reasonable given the large sample size used in this study. Inbreeding could be accounted for by the use of nine condensed Jacquard coefficients (Hanghoj et al., 2019, Jacquard, 1974) in the development of the likelihood ratio. Inbreeding could yield other levels of relationship in-between FS, 3/4S, and 2nd degree. The $\left(\hat{k}_{0}, \hat{k}_{1}\right)$-plot of the GCAT data in Fig. 4 reveal closeness of the $3 / 4 \mathrm{~S}$ and FS pairs, and suggests intermediate relationships like seven-eighths siblings (7/8S) might also exist in the data. Indeed, the full range of $2 \mathrm{ND}, 5 / 8 \mathrm{~S}, 3 / 4 \mathrm{~S}$, $7 / 8 \mathrm{~S}$, and FS relationships could be present in the data. It is easily shown that $5 / 8 \mathrm{~S}$ and $7 / 8 \mathrm{~S}$ have a kinship coefficient of 5/32 and 7/32, respectively. Figure 4 also shows evidence of some pairs in-between a 2 nd a 3rd-degree relationship. In future work, the likelihood ratio approach presented in this paper could be further refined to identify all these relationships more precisely. In-between relationships, like the $3 / 4 \mathrm{~S}$ relationship studied in this paper, essentially stress that relatedness is a continuous rather than a discrete concept. 
Table 4 Likelihood ratio inference (LR approach) for the presumably 2nd, 3/4S, and FS pairs from the GCAT cohort.

\begin{tabular}{|c|c|c|c|c|c|c|c|c|c|c|c|c|c|}
\hline Pair & IID & Sex & IID & Sex & $\hat{k}_{0}$ & $\hat{k}_{1}$ & $\hat{k}_{2}$ & $\hat{\phi}$ & LR-kinbiplot & FS UN & 3/4S UN & 2nd $\sim \mathrm{UN}$ & LR approach \\
\hline 1 & REL_00178 & F & REL_01132 & F & 0.61 & 0.36 & 0.04 & 0.107 & 2nd & -0.0165 & 0.0027 & 0.0092 & 2nd \\
\hline 2 & REL_02227 & $\mathrm{F}$ & REL_00865 & M & 0.57 & 0.43 & 0.00 & 0.109 & 2 nd & -0.0164 & 0.0035 & 0.0109 & 2nd \\
\hline 3 & REL_04137 & F & REL_03163 & M & 0.51 & 0.49 & 0.00 & 0.122 & 2nd & -0.0103 & 0.0082 & 0.0142 & 2nd \\
\hline 4 & REL_04126 & $\mathrm{F}$ & REL_02089 & $\mathrm{F}$ & 0.50 & 0.50 & 0.00 & 0.126 & $2 \mathrm{nd}$ & -0.0106 & 0.0080 & 0.0143 & $2 \mathrm{nd}$ \\
\hline 5 & REL_04141 & $\mathrm{F}$ & REL_02030 & M & 0.49 & 0.50 & 0.01 & 0.129 & $2 \mathrm{nd}$ & -0.0072 & 0.0101 & 0.0152 & $2 \mathrm{nd}$ \\
\hline 6 & REL_02092 & M & REL_00587 & $\mathrm{F}$ & 0.48 & 0.52 & 0.00 & 0.129 & $2 \mathrm{nd}$ & -0.0073 & 0.0104 & 0.0158 & $2 \mathrm{nd}$ \\
\hline 7 & REL_02212 & M & REL_04828 & F & 0.47 & 0.53 & 0.00 & 0.132 & $2 \mathrm{nd}$ & -0.0061 & 0.0111 & 0.0161 & $2 \mathrm{nd}$ \\
\hline 8 & REL_00603 & $\mathrm{F}$ & REL_00189 & F & 0.47 & 0.53 & 0.00 & 0.134 & 2nd & -0.0076 & 0.0101 & 0.0156 & 2nd \\
\hline 9 & REL_03666 & M & REL_02902 & M & 0.47 & 0.53 & 0.00 & 0.134 & 2 nd & -0.0057 & 0.0112 & 0.0160 & 2nd \\
\hline 10 & REL_00132 & $\mathrm{F}$ & REL_00707 & M & 0.45 & 0.55 & 0.00 & 0.137 & 2nd & -0.0059 & 0.0113 & 0.0164 & 2nd \\
\hline 11 & REL_02058 & $\mathrm{F}$ & REL_03610 & $\mathrm{F}$ & 0.45 & 0.55 & 0.00 & 0.139 & $2 \mathrm{nd}$ & -0.0041 & 0.0125 & 0.0170 & $2 \mathrm{nd}$ \\
\hline 12 & REL_01692 & $\mathrm{F}$ & REL_00010 & $\mathrm{F}$ & 0.44 & 0.56 & 0.00 & 0.139 & $2 \mathrm{nd}$ & -0.0041 & 0.0127 & 0.0173 & 2nd \\
\hline 13 & REL_03969 & M & REL_00271 & M & 0.34 & 0.56 & 0.10 & 0.189 & $3 / 4 \mathrm{~S}$ & 0.0260 & 0.0328 & 0.0279 & $3 / 4 \mathrm{~S}$ \\
\hline 14 & REL_03803 & $\mathrm{F}$ & REL_02343 & M & 0.35 & 0.51 & 0.14 & 0.198 & $3 / 4 \mathrm{~S}$ & 0.0317 & 0.0361 & 0.0287 & $3 / 4 \mathrm{~S}$ \\
\hline 15 & REL_03924 & M & REL_03023 & $\mathrm{F}$ & 0.37 & 0.46 & 0.17 & 0.201 & $3 / 4 \mathrm{~S}$ & 0.0365 & 0.0393 & 0.0301 & $3 / 4 \mathrm{~S}$ \\
\hline 16 & REL_00083 & M & REL_02333 & M & 0.33 & 0.52 & 0.15 & 0.207 & $3 / 4 \mathrm{~S}$ & 0.0377 & 0.0403 & 0.0313 & $3 / 4 \mathrm{~S}$ \\
\hline 17 & REL_01344 & M & REL_02408 & $\mathrm{F}$ & 0.36 & 0.44 & 0.20 & 0.210 & $3 / 4 \mathrm{~S}$ & 0.0402 & 0.0412 & 0.0304 & $3 / 4 \mathrm{~S}$ \\
\hline 18 & REL_04189 & M & REL_00775 & M & 0.36 & 0.44 & 0.20 & 0.210 & $3 / 4 \mathrm{~S}$ & 0.0422 & 0.0428 & 0.0314 & $3 / 4 \mathrm{~S}$ \\
\hline 19 & REL_03150 & $\mathrm{F}$ & REL_01804 & $\mathrm{F}$ & 0.32 & 0.51 & 0.17 & 0.212 & $3 / 4 \mathrm{~S}$ & 0.0411 & 0.0426 & 0.0322 & $3 / 4 \mathrm{~S}$ \\
\hline 20 & REL_02752 & $\mathrm{F}$ & REL_04859 & $\mathrm{F}$ & 0.34 & 0.46 & 0.20 & 0.215 & $3 / 4 \mathrm{~S}$ & 0.0441 & 0.0443 & 0.0325 & $3 / 4 \mathrm{~S}$ \\
\hline 21 & REL_01502 & M & REL_03665 & M & 0.31 & 0.48 & 0.21 & 0.225 & FS & 0.0482 & 0.0469 & 0.0339 & FS \\
\hline 22 & REL_04592 & $\mathrm{F}$ & REL_04600 & $\mathrm{F}$ & 0.30 & 0.48 & 0.21 & 0.226 & FS & 0.0511 & 0.0493 & 0.0358 & FS \\
\hline 23 & REL_04693 & $\mathrm{F}$ & REL_00797 & $\mathrm{F}$ & 0.31 & 0.47 & 0.22 & 0.228 & FS & 0.0520 & 0.0498 & 0.0357 & FS \\
\hline 24 & REL_03607 & M & REL_00319 & F & 0.30 & 0.49 & 0.21 & 0.228 & FS & 0.0501 & 0.0484 & 0.0350 & FS \\
\hline 25 & REL_03220 & $\mathrm{F}$ & REL_04615 & $\mathrm{F}$ & 0.31 & 0.46 & 0.23 & 0.230 & FS & 0.0532 & 0.0505 & 0.0360 & FS \\
\hline 26 & REL_03212 & M & REL_02516 & $\mathrm{F}$ & 0.28 & 0.53 & 0.20 & 0.231 & FS & 0.0548 & 0.0526 & 0.0386 & FS \\
\hline 27 & REL_03310 & M & REL_03659 & F & 0.26 & 0.56 & 0.18 & 0.231 & FS & 0.0496 & 0.0484 & 0.0358 & FS \\
\hline 28 & REL_04427 & $\mathrm{F}$ & REL_02635 & $\mathrm{F}$ & 0.26 & 0.54 & 0.19 & 0.232 & FS & 0.0502 & 0.0487 & 0.0358 & FS \\
\hline 29 & REL_00122 & M & REL_01902 & $\mathrm{F}$ & 0.29 & 0.49 & 0.22 & 0.233 & FS & 0.0542 & 0.0513 & 0.0368 & FS \\
\hline 30 & REL_00284 & M & REL_02444 & $\mathrm{F}$ & 0.28 & 0.51 & 0.21 & 0.233 & FS & 0.0517 & 0.0494 & 0.0356 & FS \\
\hline 31 & REL_03838 & $\mathrm{F}$ & REL_02496 & F & 0.31 & 0.45 & 0.24 & 0.234 & FS & 0.0561 & 0.0523 & 0.0367 & FS \\
\hline 32 & REL_01564 & $\mathrm{F}$ & REL_03827 & $\mathrm{F}$ & 0.32 & 0.43 & 0.26 & 0.236 & FS & 0.0571 & 0.0528 & 0.0365 & FS \\
\hline 33 & REL_04529 & F & REL_04492 & F & 0.28 & 0.50 & 0.22 & 0.236 & FS & 0.0555 & 0.0522 & 0.0373 & FS \\
\hline 34 & REL_04494 & M & REL_00931 & M & 0.28 & 0.49 & 0.23 & 0.237 & FS & 0.0560 & 0.0525 & 0.0373 & FS \\
\hline 35 & REL_04466 & $\mathrm{F}$ & REL_02680 & $\mathrm{F}$ & 0.31 & 0.43 & 0.26 & 0.237 & FS & 0.0576 & 0.0531 & 0.0367 & FS \\
\hline 36 & REL_04405 & M & REL_03949 & M & 0.26 & 0.52 & 0.22 & 0.238 & FS & 0.0557 & 0.0525 & 0.0376 & FS \\
\hline 37 & REL_03880 & M & REL_04789 & $\mathrm{F}$ & 0.27 & 0.50 & 0.23 & 0.239 & FS & 0.0566 & 0.0529 & 0.0376 & FS \\
\hline 38 & REL_00383 & $\mathrm{F}$ & REL_03293 & M & 0.25 & 0.53 & 0.22 & 0.241 & FS & 0.0574 & 0.0538 & 0.0385 & FS \\
\hline 39 & REL_01888 & M & REL_04360 & M & 0.25 & 0.54 & 0.21 & 0.241 & FS & 0.0566 & 0.0532 & 0.0383 & FS \\
\hline 40 & REL_00792 & $\mathrm{F}$ & REL_00954 & M & 0.26 & 0.51 & 0.23 & 0.242 & FS & 0.0585 & 0.0543 & 0.0385 & FS \\
\hline 41 & REL_00872 & $\mathrm{F}$ & REL_01784 & $\mathrm{F}$ & 0.25 & 0.53 & 0.22 & 0.242 & FS & 0.0598 & 0.0556 & 0.0398 & FS \\
\hline 42 & REL_01450 & M & REL_01960 & M & 0.26 & 0.51 & 0.23 & 0.242 & FS & 0.0586 & 0.0544 & 0.0386 & FS \\
\hline 43 & REL_04616 & $\mathrm{F}$ & REL_02777 & $\mathrm{F}$ & 0.28 & 0.47 & 0.25 & 0.243 & FS & 0.0604 & 0.0553 & 0.0386 & FS \\
\hline 44 & REL_02899 & M & REL_01707 & $\mathrm{F}$ & 0.28 & 0.45 & 0.26 & 0.244 & FS & 0.0618 & 0.0562 & 0.0389 & FS \\
\hline 45 & REL_02905 & $\mathrm{F}$ & REL_02575 & $\mathrm{F}$ & 0.25 & 0.52 & 0.23 & 0.245 & FS & 0.0604 & 0.0557 & 0.0394 & FS \\
\hline 46 & REL_00769 & M & REL_04746 & $\mathrm{F}$ & 0.23 & 0.57 & 0.21 & 0.246 & FS & 0.0606 & 0.0564 & 0.0406 & FS \\
\hline 47 & REL_00009 & $\mathrm{F}$ & REL_02335 & $\mathrm{F}$ & 0.23 & 0.55 & 0.22 & 0.246 & FS & 0.0603 & 0.0558 & 0.0399 & FS \\
\hline 48 & REL_04475 & $\mathrm{F}$ & REL_04218 & M & 0.25 & 0.51 & 0.24 & 0.247 & FS & 0.0615 & 0.0564 & 0.0397 & FS \\
\hline 49 & REL_01150 & $\mathrm{F}$ & REL_04384 & $\mathrm{F}$ & 0.26 & 0.49 & 0.25 & 0.249 & FS & 0.0639 & 0.0580 & 0.0403 & FS \\
\hline 50 & REL_03944 & M & REL_03475 & $\mathrm{F}$ & 0.23 & 0.54 & 0.23 & 0.249 & FS & 0.0618 & 0.0568 & 0.0403 & FS \\
\hline 51 & REL_03904 & $\mathrm{F}$ & REL_04994 & $\mathrm{F}$ & 0.25 & 0.50 & 0.25 & 0.249 & FS & 0.0631 & 0.0573 & 0.0400 & FS \\
\hline 52 & REL_01654 & M & REL_03485 & M & 0.28 & 0.43 & 0.29 & 0.251 & FS & 0.0660 & 0.0588 & 0.0398 & FS \\
\hline 53 & REL_00504 & M & REL_04718 & $\mathrm{F}$ & 0.24 & 0.50 & 0.25 & 0.252 & FS & 0.0645 & 0.0582 & 0.0404 & FS \\
\hline 54 & REL_00339 & $\mathrm{F}$ & REL_02473 & $\mathrm{F}$ & 0.25 & 0.48 & 0.27 & 0.253 & FS & 0.0651 & 0.0584 & 0.0400 & FS \\
\hline 55 & REL_01016 & M & REL_00887 & M & 0.24 & 0.50 & 0.26 & 0.254 & FS & 0.0661 & 0.0594 & 0.0411 & FS \\
\hline 56 & REL_03977 & M & REL_01080 & M & 0.22 & 0.54 & 0.24 & 0.255 & FS & 0.0644 & 0.0583 & 0.0408 & FS \\
\hline
\end{tabular}


Table 4 (continued)

\begin{tabular}{|c|c|c|c|c|c|c|c|c|c|c|c|c|c|}
\hline Pair & IID & Sex & IID & Sex & $\hat{k}_{0}$ & $\hat{k}_{1}$ & $\hat{k}_{2}$ & $\hat{\phi}$ & LR-kinbiplot & FS UN & $3 / 4 \mathrm{~S} \sim \mathrm{UN}$ & 2nd $\sim \mathrm{UN}$ & LR approach \\
\hline 57 & REL_02339 & M & REL_02391 & M & 0.27 & 0.44 & 0.29 & 0.256 & FS & 0.0688 & 0.0608 & 0.0411 & FS \\
\hline 58 & REL_01524 & $\mathrm{F}$ & REL_03272 & $\mathrm{F}$ & 0.23 & 0.51 & 0.26 & 0.256 & FS & 0.0674 & 0.0604 & 0.0419 & FS \\
\hline 59 & REL_01285 & M & REL_03761 & F & 0.24 & 0.50 & 0.27 & 0.257 & FS & 0.0670 & 0.0597 & 0.0410 & FS \\
\hline 60 & REL_03395 & $\mathrm{F}$ & REL_02694 & F & 0.22 & 0.52 & 0.25 & 0.257 & FS & 0.0680 & 0.0609 & 0.0423 & FS \\
\hline 61 & REL_03151 & M & REL_02204 & $\mathrm{F}$ & 0.23 & 0.50 & 0.26 & 0.257 & FS & 0.0683 & 0.0610 & 0.0421 & FS \\
\hline 62 & REL_00968 & M & REL_01577 & F & 0.26 & 0.45 & 0.29 & 0.259 & FS & 0.0744 & 0.0654 & 0.0445 & FS \\
\hline 63 & REL_04439 & $\mathrm{F}$ & REL_01640 & F & 0.26 & 0.43 & 0.31 & 0.260 & FS & 0.0721 & 0.0630 & 0.0421 & FS \\
\hline 64 & REL_01546 & M & REL_03566 & F & 0.21 & 0.53 & 0.26 & 0.263 & FS & 0.0701 & 0.0621 & 0.0428 & FS \\
\hline 65 & REL_03442 & $\mathrm{F}$ & REL_04510 & $\mathrm{F}$ & 0.22 & 0.51 & 0.27 & 0.264 & FS & 0.0714 & 0.0630 & 0.0431 & FS \\
\hline 66 & REL_00340 & F & REL_04294 & F & 0.21 & 0.53 & 0.26 & 0.264 & FS & 0.0710 & 0.0628 & 0.0432 & FS \\
\hline 67 & REL_03001 & $\mathrm{F}$ & REL_04111 & $\mathrm{F}$ & 0.23 & 0.48 & 0.29 & 0.265 & FS & 0.0727 & 0.0636 & 0.0430 & FS \\
\hline 68 & REL_00282 & $\mathrm{F}$ & REL_04918 & $\mathrm{F}$ & 0.25 & 0.44 & 0.31 & 0.267 & FS & 0.0748 & 0.0648 & 0.0430 & FS \\
\hline 69 & REL_01083 & $\mathrm{F}$ & REL_01704 & $\mathrm{F}$ & 0.18 & 0.57 & 0.25 & 0.267 & FS & 0.0715 & 0.0634 & 0.0439 & FS \\
\hline 70 & REL_03388 & $\mathrm{F}$ & REL_02608 & $\mathrm{F}$ & 0.22 & 0.50 & 0.29 & 0.268 & FS & 0.0739 & 0.0645 & 0.0436 & FS \\
\hline 71 & REL_01924 & $\mathrm{F}$ & REL_00727 & M & 0.24 & 0.45 & 0.32 & 0.270 & FS & 0.0769 & 0.0663 & 0.0440 & FS \\
\hline 72 & REL_02208 & F & REL_03486 & $\mathrm{F}$ & 0.23 & 0.46 & 0.31 & 0.270 & FS & 0.0769 & 0.0665 & 0.0444 & FS \\
\hline 73 & REL_02718 & M & REL_02913 & M & 0.22 & 0.48 & 0.30 & 0.271 & FS & 0.0765 & 0.0662 & 0.0443 & FS \\
\hline 74 & REL_00634 & M & REL_03507 & M & 0.20 & 0.51 & 0.29 & 0.272 & FS & 0.0754 & 0.0656 & 0.0443 & FS \\
\hline 75 & REL_04741 & $\mathrm{F}$ & REL_02513 & $\mathrm{F}$ & 0.19 & 0.52 & 0.30 & 0.277 & FS & 0.0783 & 0.0676 & 0.0455 & FS \\
\hline 76 & REL_00601 & M & REL_02989 & $\mathrm{F}$ & 0.19 & 0.51 & 0.30 & 0.278 & FS & 0.0802 & 0.0689 & 0.0462 & FS \\
\hline 77 & REL_01624 & $\mathrm{F}$ & REL_00750 & $\mathrm{F}$ & 0.19 & 0.51 & 0.30 & 0.278 & FS & 0.0790 & 0.0680 & 0.0456 & FS \\
\hline 78 & REL_00824 & $\mathrm{F}$ & REL_00213 & $\mathrm{F}$ & 0.22 & 0.45 & 0.33 & 0.278 & FS & 0.0815 & 0.0693 & 0.0456 & FS \\
\hline 79 & REL_01264 & M & REL_04751 & F & 0.18 & 0.52 & 0.30 & 0.279 & FS & 0.0795 & 0.0684 & 0.0459 & FS \\
\hline 80 & REL_02208 & F & REL_01630 & F & 0.18 & 0.52 & 0.31 & 0.283 & FS & 0.0826 & 0.0706 & 0.0473 & FS \\
\hline 81 & REL_04704 & $\mathrm{F}$ & REL_00804 & M & 0.17 & 0.52 & 0.31 & 0.285 & FS & 0.0829 & 0.0707 & 0.0472 & FS \\
\hline 82 & REL_03627 & $\mathrm{F}$ & REL_03315 & $\mathrm{F}$ & 0.15 & 0.55 & 0.30 & 0.288 & FS & 0.0838 & 0.0714 & 0.0478 & FS \\
\hline 83 & REL_03486 & $\mathrm{F}$ & REL_01630 & $\mathrm{F}$ & 0.17 & 0.50 & 0.33 & 0.289 & FS & 0.0873 & 0.0738 & 0.0488 & FS \\
\hline
\end{tabular}

FS UN, 3/4S UN and 2nd UN are the LR values for each pair. LR-kinbiplot is the inferred relationship from Graffelman et al. (2019). $\hat{\phi}$ : estimated kinship coefficient. $\hat{k}_{0}, \hat{k}_{1}$, and $\hat{k}_{2}$ : estimated IBD probabilities.

Maximum values of the likelihood ratios of each pair are marked in bold.
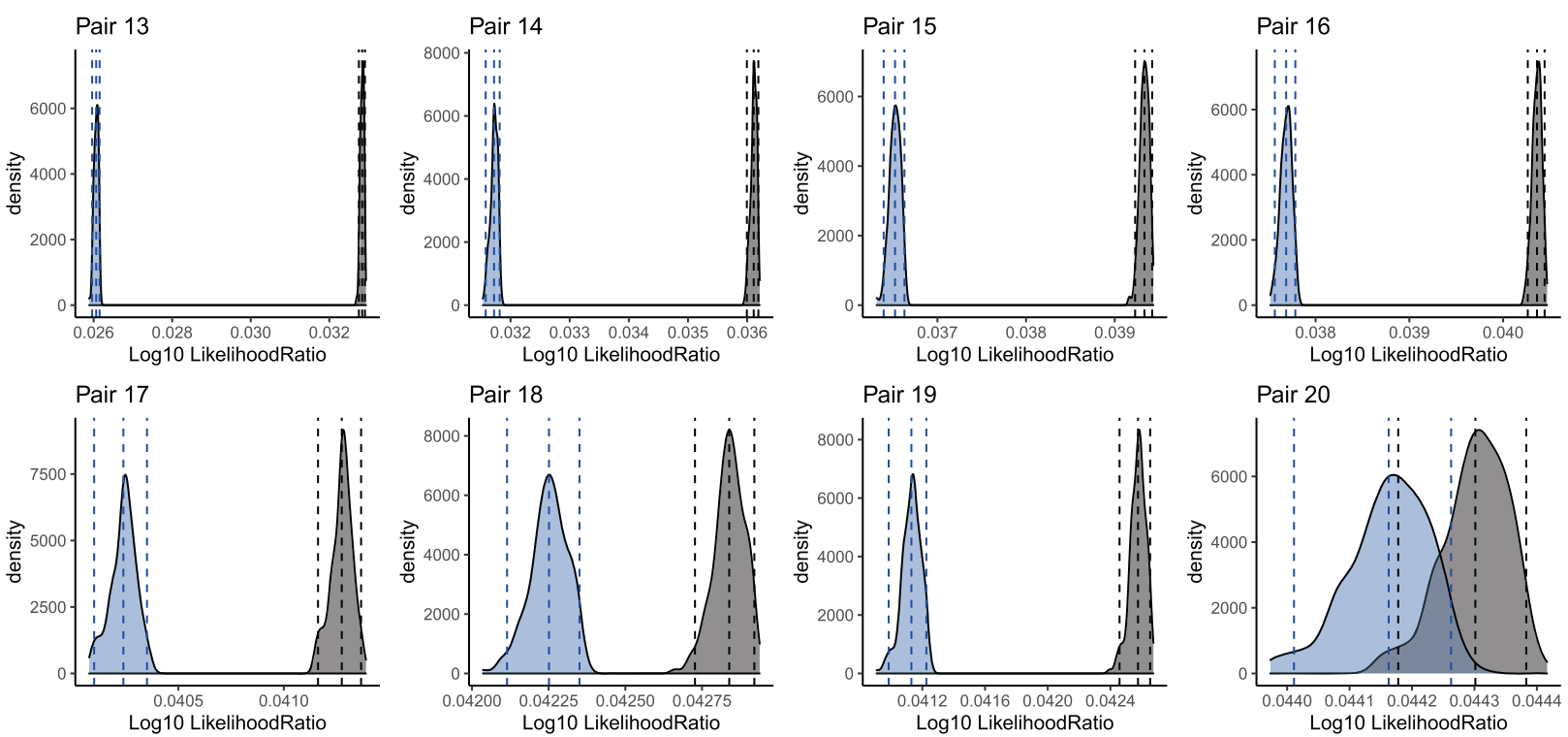

Fig. 7 Bootstrap distribution of the LR for eight presumably 3/4S pairs of the GCAT project. Vertical dashed lines indicate the average LR values and the $95 \%$ bootstrap confidence interval limits. 
Acknowledgements This study makes use of data generated by the GCAT Genomes for Life Cohort study of the Genomes of Catalonia, IGTP. A full list of the investigators who contributed to the generation of the data is available from www.genomesforlife.com. We thank the CERCA Program of the Generalitat de Catalunya for institutional support. We are also very grateful to Bruce S. Weir for his comments on the manuscript as well as the computer resources and technical expertize provided by Daniel Matías-Sánchez, Jordi Valls-Margarit, and David Torrents-Arenales from the Life Sciences-Computational Genomics group of the Barcelona Supercomputing Center - Centro Nacional de Supercomputación (BSC-CNS).

Funding This work was partially supported by grants RTI2018095518-B-C22 (JG), RTI2018-095518-B-C21 (IGF and CBV), and ADE 10/00026 (RdC) (MCIU/AEI/FEDER) of the Spanish Ministry of Science, Innovation and Universities and the European Regional Development Fund, by grants SGR1269 and 2017 SGR529 (RdC) of the Generalitat de Catalunya, by grant R01 GM075091 (JG) from the United States National Institutes of Health, by the Ramon y Cajal action RYC-2011-07822 (RdC), by Agency for Management of University and Research Grants (AGAUR) of the Catalan Government grant 2017SGR723 (VM), and by the Spanish Association Against Cancer (AECC) Scientific Foundation, grant GCTRA18022MORE (VM).

\section{Compliance with ethical standards}

Conflict of interest The authors declare that they have no conflict of interest.

Publisher's note Springer Nature remains neutral with regard to jurisdictional claims in published maps and institutional affiliations.

Open Access This article is licensed under a Creative Commons Attribution 4.0 International License, which permits use, sharing, adaptation, distribution and reproduction in any medium or format, as long as you give appropriate credit to the original author(s) and the source, provide a link to the Creative Commons license, and indicate if changes were made. The images or other third party material in this article are included in the article's Creative Commons license, unless indicated otherwise in a credit line to the material. If material is not included in the article's Creative Commons license and your intended use is not permitted by statutory regulation or exceeds the permitted use, you will need to obtain permission directly from the copyright holder. To view a copy of this license, visit http://creativecommons. org/licenses/by/4.0/.

\section{Appendix}

\section{Appendix A}

We derive the IBD probabilities for three-quarter siblings $(3 / 4 \mathrm{~S})$ in the case that a pair of individuals has one parent in common while their unshared parents are full siblings (FS) (Fig. 8). In the case that the unshared parents have a parent-offspring relationship, the IBD probabilities can be derived analogously.

Let $\delta \gamma$ be the genotype of the common parent of a $3 / 4 \mathrm{~S}$ pair, and $\alpha \beta, \alpha B, A \beta$, and $A B$ the possible genotypes of an
FS pair. Then, all the possible genotypes and the IBD alleles shared for a $3 / 4 \mathrm{~S}$ pair are shown in Table 5 .

From Table 5, the IBD probabilities for $3 / 4 \mathrm{~S}$ are:

$$
\begin{aligned}
& k_{0}=P(I B D=0)=\frac{24}{64}=3 / 8 \\
& k_{1}=P(I B D=1)=\frac{32}{64}=1 / 2 \\
& k_{2}=P(I B D=2)=\frac{8}{64}=1 / 8
\end{aligned}
$$

And their kinship coefficient is:

$$
\phi=k_{1} / 4+k_{2} / 2=\frac{1}{2} \frac{1}{4}+\frac{1}{8} \frac{1}{2}=3 / 16
$$

\section{Appendix B}

Here we show the LR of 3/4S UN for a biallelic SNP whose alleles are $A$ and $B$. Let $p$ and $q$ be the allele frequencies for $A$ and $B$ of the population under study. For a pair of individuals, we show the LR computation for four genotype pairs: $A A / A A$, $A A / A B, A A / B B$ and $A B / A B$. The LR for the remaining genotype pairs $(A B / A A, A B / B B, B B / A A, B B / A B$, and $B B / B B)$ are equivalent or can be obtained analogously.

The IBD probabilities for $3 / 4 \mathrm{~S}$ are $\left(k_{0}, k_{1}, k_{2}\right)=(3 / 8,1 / 2$, $1 / 8)$ and for UN pairs are $\left(k_{0}, k_{1}, k_{2}\right)=(1,0,0)$. Then, according to Tables 1 and 2 and Eqs (1) and (2), the LR for $3 / 4 \mathrm{~S} \sim \mathrm{UN}$ is derived as follows:

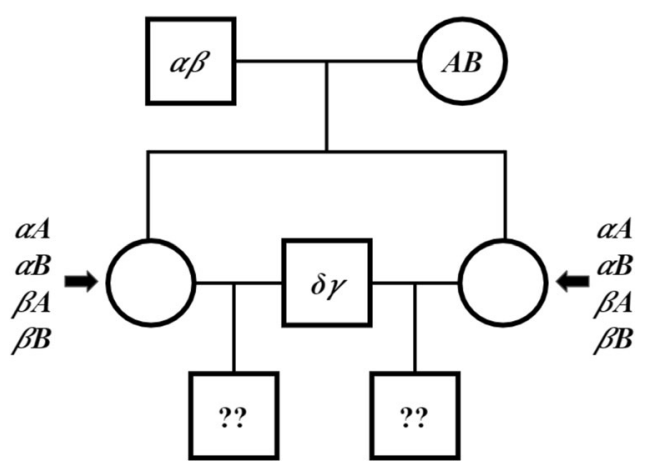

Fig. 8 Pedigree of a 3/4S pair where their unshared parents are FS.

Table 5 Number of IBD alleles for all possible pairs of 3/4S where their unshared parents are FS.

\begin{tabular}{lllllllll}
\hline & $\alpha \delta$ & $\alpha \gamma$ & $A \delta$ & $A \gamma$ & $\beta \delta$ & $\beta \gamma$ & $B \delta$ & $B \gamma$ \\
\hline$\alpha \delta$ & 2 & 1 & 1 & 0 & 1 & 0 & 1 & 0 \\
$\alpha \gamma$ & 1 & 2 & 0 & 1 & 0 & 1 & 0 & 1 \\
$A \delta$ & 1 & 0 & 2 & 1 & 1 & 0 & 1 & 0 \\
$A \gamma$ & 0 & 1 & 1 & 2 & 0 & 1 & 0 & 1 \\
$\beta \delta$ & 1 & 0 & 1 & 0 & 2 & 1 & 1 & 0 \\
$\beta \gamma$ & 0 & 1 & 0 & 1 & 1 & 2 & 0 & 1 \\
$B \delta$ & 1 & 0 & 1 & 0 & 1 & 0 & 2 & 1 \\
$B \gamma$ & 0 & 1 & 0 & 1 & 0 & 1 & 1 & 2 \\
\hline
\end{tabular}




\section{$A A / A A$ case:}

$L R=\frac{\frac{3}{8} p^{4}+\frac{1}{2} p^{3}+\frac{1}{8} p^{2}}{p^{4}}=\frac{3}{8}+\frac{1}{2 p}+\frac{1}{8 p^{2}}$

$A A / A B$ case:

$L R=\frac{\frac{3}{8} 2 p^{3} q+\frac{1}{2} p^{2} q}{2 p^{3} q}=\frac{3}{8}+\frac{1}{4 p}$

\section{$A A / B B$ case:}

$L R=\frac{\frac{3}{8} p^{2} q^{2}}{p^{2} q^{2}}=\frac{3}{8}$

\section{$A B / A B$ case:}

$L R=\frac{\frac{3}{8} 4 p^{2} q^{2}+\frac{1}{2} p q+\frac{1}{8} 2 p q}{4 p^{2} q^{2}}=\frac{3}{8}+\frac{3}{16 p q}$

\section{References}

Abecasis GR, Wigginton JE (2005) Handling marker-marker linkage disequilibrium: pedigree analysis with clustered markers. Am J Hum Genet 77:754-767

Abecasis GR, Cherny SS, Cookson WOC, Cardon LR (2001) GRR: graphical representation of relationship errors. Bioinformatics 17:742-743

Anderson CA, Pettersson FH, Clarke GM, Cardon LR, Morris AP, Zondervan KT (2010) Data quality control in genetic case-control association studies. Nat Protoc 5:1564

Bhérer C, Campbell CL, Auton A (2017) Refined genetic maps reveal sexual dimorphism in human meiotic recombination at multiple scales. Nat Commun 8:1-9

Boehnke M, Cox NJ (1997) Accurate inference of relationships in sibpair linkage studies. Am J Hum Genet 61:423-429

Bycroft C, Freeman C, Petkova D, Band G, Elliott LT, Sharp K et al. (2018) The UK Biobank resource with deep phenotyping and genomic data. Nature 562:203

Caballero M, Seidman DN, Qiao Y, Sannerud J, Dyer TD, Lehman DM et al. (2019) Crossover interference and sex-specific genetic maps shape identical by descent sharing in close relatives. PLoS Genet 15:e1007979

Calus MPL, Vandenplas J (2018) SNPrune: an efficient algorithm to prune large SNP array and sequence datasets based on high linkage disequilibrium. Genet Selection Evolution 50:34

Campbell CL, Furlotte NA, Eriksson N, Hinds D, Auton A (2015) Escape from crossover interference increases with maternal age. Nat Commun 6:6260

Delaneau O, Zagury J-F, Robinson MR, Marchini JL, Dermitzakis ET (2019) Accurate, scalable and integrative haplotype estimation. Nat Commun 10:1-10

Efron B (1994) Tibshirani RJ. An introduction to the bootstrap. CRC press

Evett IW, Weir BS (1998) Interpreting DNA evidence. Sinauer Associates, Inc

Galván-Femenía I, Graffelman J, Barceló-Vidal C (2017) Graphics for relatedness research. Mol Ecol Resour 17:1271-1282
Galván-Femenía I, Obón-Santacana M, Piñeyro D, Guindo-Martinez M, Duran X, Carreras A et al. (2018) Multitrait genome association analysis identifies new susceptibility genes for human anthropometric variation in the GCAT cohort. J Med Genet 55:765-778

Goudet J, Kay T, Weir BS (2018) How to estimate kinship. Mol Ecol 27:4121-4135

Graffelman J, Galván-Femenía I, de Cid R, Barceló-Vidal C (2019) A log-ratio biplot approach for exploring genetic relatedness based on identity by state. Front Genet 10:341

Graffelman J, Moreno V (2013) The mid p-value in exact tests for HardyWeinberg equilibrium. Stat Appl Genet Mol Biol 12:433-448

Hanghøj K, Moltke I, Andersen PA, Manica A, Korneliussen TS (2019) Fast and accurate relatedness estimation from high-throughput sequencing data in the presence of inbreeding. GigaScience 8:5

Heinrich V, Kamphans T, Mundlos S, Robinson PN, Krawitz PM (2016) A likelihood ratio-based method to predict exact pedigrees for complex families from next-generation sequencing data. Bioinformatics 33:72-78

Hill WG, Weir BS (2011) Variation in actual relationship as a consequence of mendelian sampling and linkage. Genet Res (Camb) 93:47-64

Jacquard A (1974) The genetic structure of populations. Springer-Verlag Karczewski KJ, Francioli LC, Tiao G, Cummings BB, Alföldi J, Wang $\mathrm{Q}$ et al. (2020) The mutational constraint spectrum quantified from variation in 141,456 humans. Nature 581:434-443

Katki HA, Sanders CL, Graubard BI, Bergen AW (2010) Using DNA fingerprints to infer familial relationships within NHANES III households. J Am Stat Assoc 105:552-563

Kling D, Tillmar A (2019) Forensic genealogy-a comparison of methods to infer distant relationships based on dense SNP data. Forensic Sci Int Genet 42:113-124

Kling D, Tillmar A, Egeland T, Mostad P (2015) A general model for likelihood computations of genetic marker data accounting for linkage, linkage disequilibrium, and mutations. Int J Leg Med 129:943-954

Manichaikul A, Mychaleckyj JC, Rich SS, Daly K, Sale M, Chen Wei-Min (2010) Robust relationship inference in genome-wide association studies. Bioinformatics 26:2867-2873

Milligan BG (2003) Maximum-likelihood estimation of relatedness. Genetics 163:1153-1167

Mo SK, Liu Y-C, Wang S-Q, Bo X-C, Li Z, Chen Y et al. (2016) Exploring the efficacy of paternity and kinship testing based on single nucleotide polymorphisms. Forensic Sci Int Genet 22:161-168

Obón-Santacana M, Vilardell M, Carreras A, Duran X, Velasco J, Galván-Femenía I et al. (2018) GCATIGenomes for life: a prospective cohort study of the genomes of Catalonia. BMJ Open 8: e018324

Oliehoek PA, Windig JJ, van Arendonk JAM, Bijma P (2006) Estimating relatedness between individuals in general populations with a focus on their use in conservation programs. Genetics 173:483-496

Purcell S, Neale B, Todd-Brown K, Thomas L, Ferreira MA, Bender D et al. (2007) PLINK: a tool set for whole-genome association and population-based linkage analyses. Am J Hum Genet 81:559-575

Rosenberg NA (2006) Standardized subsets of the hgdp-ceph human genome diversity cell line panel, accounting for atypical and duplicated samples and pairs of close relatives. Ann Hum Genet 70:841-847

Staples J, Maxwell EK, Gosalia N, Gonzaga-Jauregui C, Snyder C, Hawes A et al. (2018) Profiling and leveraging relatedness in a precision medicine cohort of 92,455 exomes. Am J Hum Genet 102:874-889

Staples J, Qiao D, Cho MH, Silverman EK, Genomics U, Nickerso DA et al. (2014) PRIMUS: Rapid Reconstruction of Pedigrees from Genome-wide Estimates of Identity by Descent. Am J Hum Genet 95:553-564 
Taliun D, Harris DN, Kessler MD, Carlson J, Szpiech ZA, Torres R et al. (2019) Sequencing of 53,831 diverse genomes from the NHLBI TOPMed Program. bioRxiv

Thompson EA (1975) The estimation of pairwise relationships. Ann Hum Genet 39:173-188

Thompson EA (1986) Likelihood inference of paternity. Am J Hum Genet 39:285

Thompson EA (1991) Estimation of relationships from genetic data. Handb Stat 8:255-269

Visscher PM, Wray NR, Zhang Q, Sklar P, McCarthy MI, Brown MA et al. (2017) 10 years of GWAS discovery: biology, function, and translation. Am J Hum Genet 101:5-22. ISSN 0002-9297

Wagner AP, Creel S, Kalinowski ST (2006) Estimating relatedness and relationships using microsatellite loci with null alleles. Heredity 97:336

Wang J (2004) Sibship reconstruction from genetic data with typing errors. Genetics 166:1963-1979

Weir BS, Anderson AD, Hepler AB (2006) Genetic relatedness analysis: modern data and new challenges. Nat Rev Genet 7:771

Weir BS, Goudet J (2017) A unified characterization of population structure and relatedness. Genetics 206:2085-2103 